Revista Herencia Vol. 28 (1), 25-34, 2015

Recibido 29-08-2015 Aprobado 22-09-2015

\title{
UN DIÁLOGO PENDIENTE: El PATRIMONIO POLÍtICO/CULTURAL EN LA SEDE DE la presidencia de la República de Costa Rica (1980-2013)
}

\begin{abstract}
RESUmen
El patrimonio político y cultural de la Presidencia de la República de Costa Rica (1980-2013) constituye un eje fundamental para el devenir cívico y cultural de la comunidad costarricense y asume un papel importante para el fortalecimiento de la imagen simbólica de la nación costarricense. En este sentido, el artículo ofrece una breve exploración de las raíces coloniales que permanecen implícitas en el binomio político/cultural de la sede del gobierno, así como rasgos de la herencia eclesiástica que permanece oculta en el concepto de "patrimonio"; seguidamente, se ofrece un estudio más amplio del edificio de la sede del gobierno, mientras que se realiza un recorrido más breve -pero no menos importante- de los recursos simbólicos tangibles e intangibles presentes en la sede de la Presidencia; finalmente, se hace referencia a una serie de recomendaciones para la gestión administrativa y difusión educativa del conjunto de este patrimonio.
\end{abstract}

Palabras claves: patrimonio cultural, patrimonio político, gobierno, Presidencia, Costa Rica.

Kirsten

Grünberg.

Colaboró como curadora de arte voluntaria en la Fundación para el Rescate y

la Protección del Patrimonio de la Casa Presidencial. Posee una Maestría en Educación, realizó estudios en el Doctorado en Estudios de la Sociedad y la Cultura de la UCR y en Pratt Institute de Nueva York. Su experiencia profesional incluye el Metropolitan Museum of Art y la colaboración permanente en el Committee on Teaching About the United Nations (CTAUN).

kike.grunberg@gmail.com

\section{Abstract}

The political and cultural heritage of the Presidential Building of the Republic of Costa Rica (1980-2013) is percieved as a symbolic resource that reinforces the country's national identity. This work briefly uncovers the different forms of colonialism that remain within the political and cultural spheres of the government's site, as well as the ecclesiastical roots that act as a potential influence in the concept "heritage". The work wishes to explore the historic and symbolic interpretations associated with the building, and aims to draw up a brief inventory of the tangible and intangible heritage embraced within the government's site. Management practices of the symbolic assets, as well as the design and implementation of education resources, are also provided as recommendations.

Keywords: cultural heritage, political heritage, government, Presidential Building, Costa Rica.

\section{La presidencia de la República de Costa Rica: el patrimonio y su contexto político/cultural}

Se concibe la Presidencia de la República de Costa Rica -conocida como la Casa Presidencial-como la sede oficial del Poder Ejecutivo, donde el presidente y los representantes de su gabinete aplican las leyes enunciadas en la Constitución Política y quienes, además, conducen las políticas públicas que contribuyen al desarrollo nacional. Dentro de este contexto político y administrativo, la sede de la 
Presidencia constituye uno de los símbolos emblemáticos del país, ya que representa la consolidación de un conjunto de prácticas políticas que han conducido a fortalecer el Estado democrático de Costa Rica, resultado de la participación de la comunidad civil en las urnas y de la alternancia del poder de sus gobernantes con sus transiciones graduales.

El presente artículo parte de la premisa de que el significado emblemático de la sede de la Presidencia de la República de Costa Rica se ha desarrollado a partir de un "aura "politico/cultural en el cual se enmarca su patrimonio. La referencia de un "aura" alude a la herencia eclesiástica presente en el campo politico, un tema que puede explorarse con mayor profundidad en los argumentos planteados por Benavides Barquero (2005) en su texto Relacion entre Estado e lglesia en Costa Rica a través de sus historia. Por tanto, dentro de un contexto polfico, el concepto de "aura" se

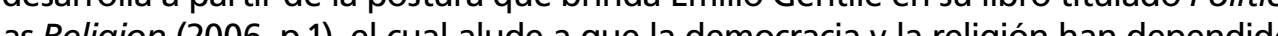
as Religion (2006, p.1), el cual alude a que la democracia y la religión han dependido de conceptos como la fe, los mitos, los ritos y los simbolos para mantener un aura sagrada y asi con ctir sus ideologias " "aja" se desprende de la teorín; y, dentro Walter Benjamin, y que Jone zación Benamin, y que Jorge Gania Torrego (201) interpe de su texto Actualzación del concepto Aura, cono una obra material que tione vigencia en un plano que se encuentran estro, en la sede de gabien es su "aura" politica, ya que la sede de la Presidre si. el pricenta, y el más evidente,

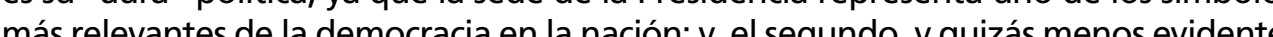
es su "aura" cultural, reflejacia en la nacion, y, el segundo, y quizás me los evidente, presentes en la sede del gobierno, incluyendo el sllo que le imprimió Pedro Ramírez presentes en la sede del gobieno, incluyendo el sello que le impinió

Es importante destacar que, además de la herencia eclesiástica, el binomio político/cultural de la Presidencia también hereda otra serie de rasgos propios del tiempo de la Colonia. De acuerdo con Quijano (2000, p.233), la población indígena, afro descendiente y mestiza no tuvo la oportunidad de tomar decisiones sociales y políticas durante la organización de los nuevos Estados en América Latina y, por tanto, aunque algunos de sus recursos simbólicos se en cuentran presentes en la sede de la Presidencia, éstos no fueron legitima en por las decisiones que tomaron estos grupos representativos de la diversidad por las decision que diversidad cultural y sos recursos de estas poblaciones fueron extraídos de sus propios contrario, los recursos de estas poblaciones fueron extraidos de sus propios por las jerarquías político/culturales que han imperado en el país.

Dentro de este contexto, el mismo deseo de conservar los recursos simbólicos que se desprenden del marco auriático político/cultural de la sede de la Presidencia, parte de este complejo entramado eclesiástico colonial. El sociólogo Henri De Man (mencionado por Gentile, 2006, p.7) afirma que "la nostalgia para unas condiciones mejores en el futuro" surge de un instinto escatológico desarrollado en la fe cristiana. Por lo tanto, es posible afirmar que el concepto de "patrimonio" que se pretende desarrollar en este artículo tiene sus limitaciones "patrimonio" que se pretende desarrollar en este artículo tiene sus limitaciones
conceptuales ya que, el fin de transmitir un conjunto de recursos simbólicos a las generaciones futuras, parece desprenderse también de las complejas raíces religiosas heredadas de la presencia colonial.

Por tanto, consciente del riesgo de aplicar el concepto de "patrimonio", el presente artículo se referirá a él ya que, hasta el momento, la autora no ha logrado identificar otro término, concepto o palabra, que pueda referirse a un conjunto de recursos simbólicos tangibles e intangibles que se enmarcan dentro de la memoria institucional de la sede de la Presidencia. La autora también está consciente de que recurre a una serie de fuentes de conocimiento y metodologías que surgen de una narrativa académica con el fin de identificar y analizar este conjunto de recursos simbólicos. Por tanto, los resultados que se identifican en este artículo deben de analizarse continuamente por grupos representativos de la diversidad cultural y social del país con el fin de afirmar, destacar, modificar o destituir su carácter "patrimonial". De esta forma y, través de su representación colectiva, se podrán aplicar las que deben de continuar presentes en "la casa de todos los costarricenses".

\section{El patrimonio de la sede de la presidencia de la república}

La sede de la Presidencia de la República comprende una serie de recursos simbólicos -políticos y culturales- que pretenden ser abordados a partir del concepto de patrimonio, el cual es definido como:

el conjunto de bienes culturales y naturales, tangibles e intangibles, generados localmente, y que una generación hereday transmite a la siguiente con el pro-

Fundación ILAM, 2015).

Por lo tanto, el carácter "patrimonial" va a tender a dirigir su mirada a todo aquello que busque protegerse, recuperarse y difundirse con el fin de que una comunidad logre reconocerse cocuperarse y dit

Con el fin de determinar los diferentes tipos de bienes con carácter patrimonial en la sede de la Presidencia, también resulta importante reconocer las diferentes categorías que propone la UNESCO (2008):

1. el patrimonio cultural material:

- el patrimonio cultural mueble (pinturas, esculturas, monedas, manuscritos, etc.);

el patrimonio cultural inmueble (monumentos, sitios arqueológicos, etc.)

- el patrimonio cultural subacuático (restos de naufragios, ruinas y ciudades sumergidas, etc);

2. el patrimonio cultural inmaterial (tradiciones y expresiones orales, incluido el idioma como vehículo del patrimonio cultural inmaterial; artes del espectáculo, como la música tradicional, la danza y el teatro; usos sociales, rituales y actos festivos; conocimientos y usos relacionados con la naturaleza y el universo; técnicas artesanales tradicionales, etc.)

3. el patrimonio natural (sitios naturales que revisten aspectos culturales como los paisajes culturales, las formaciones físicas, biológicas o geológicas, etc.)

De igual forma, el Ministerio de Cultura y Juventud (2012) brindó, en su momento, una propuesta con parámetros similares adaptados a un contexto naciolas expresiones inmateriales de la sede de la Presidencia -que se expresa dentro 
de un espacio y un tiempo histórico determinado- facilita la gestión administrativa, la documentación, la catalogación y la difusión educativa del mismo.

\section{El patrimonio político}

El patrimonio intangible de la sede de la Presidencia de la República surge de un conjunto de acontecimientos políticos, culturales y sociales que han forjado e carăcter nacional, asi como las actividades conmemorativas de diversos actos civicos que se han llevado a cabo en la sede del gobierno. Para la identificación y recopilación de este patrimonio intangible es necesario indagar los archivos de las diez 2013: Laura Chinchilla (2010-2014). Oscar Arias Sánchez (2006-2010), enel Pasode la Espriella (2002-2006) Miguel Ánge Rodríguez Echevería (1998-2002), de la Espriella (2002-2006), Miguel Angel Rodriguez ECheverra (198-2002), José Mar a Figués Olsen (1994-1998), Rafael Angel Calderón Fournier (1990-1994), Oscar Arias Sánchez (1986-19), Luis Alberto Monge Alvarez (1982-1986) y Rodrigo Carazo Odio (1978-1982). Tambien es necesario tomar en cuenta las experien

Los recursos simbolicos intangibles en el campo de la política también guardan una estrecha reloción con la herencia eclea mencionada de temporán eja contexistencial" o "espacio de vida" en donde "resuena lo divino". En un "espacio existentir de una serie de características que ofrece el autor, es posible identificar la presencia de dos "espacios sagrados" en la sede del gobierno.

1. El espacio sagrado existe gracias a la presencia de un ser superior $y$ a sus fuerzas abstractas. González (2003, p. 205) afirma que, "Lo sagrado sólo se entiende desde el ámbito de lo trascendente, es decir la relación con un mundo que sobrepasa el común de las cosas cotidianas y la posibilidad de mundo que sobreasán con un mundo superior.." Por posibilidad . patrios ubicados en diversos espacios de la sede de la Presidencia (como el Pabellón Nacional que se encuentra al frente del edificio), mientras que una fuerza abstracta lo constituye el mismo edificio que representa "la casa de todos los costarricenses", tal y como lo indica la alfombra que se encuentra en la entrada principal. En el ámbito de los representantes del gobierno, el ser superior es representado por el presidente o la presidenta de la República y su gabinete, así como por la visita de personajes del mismo rango o personajes costarricenses de prestigio. En un contexto similar, pero no menos importante, se encuentran las primeras damas y diversos
funcionarios políticos.

2. Los espacios sagrados se encuentran absolutamente estructurados y jerarquizados. González (2003, p. 261) expresa que un edificio cuenta con espacios independientes y cualitativamente diferenciados, pero que sólo se comprenden desde una unidad estructural mayor. Por tanto, los despachos de los gobrantes, los espacios públicos y una serie de oficidentro de la sede de la Presidencia.

\section{El patrimonio cultural}

Hasta la fecha, cuatro organismos principales administran el patrimonio cultural de la sede de la Presidencia: la Oficina de la Dirección General de la Presidencia (Departamento de Servicios Generales y Departamento de Activos); el Instituto Nacional de Seguros (INS); el Museo Nacional de Costa Rica y la Fundación para el ción General de la Presidencia es responsable de administrar el edificio inmueble y sus activos. Estos activos incluy ran parte del mobiliario de las oficinmueble y sus activos. Estos activos incluyen gran parte del mobiliario de las oficinas pero, las giras presidencia las giras presidenciales, nacionales entos de la Presidencia también custodia los bienes de otras instituciones gubernamende la Presidencia tambéstamo como la escultura del artista costarricense Jiménez tales, en calidad de préstamo, como la escultura del artista costarricense Jimenez Deredia que le pertenece al Instituto Nacional de Seguros (INS) (ubicada en la entrada principal de la sede de la Presidencia) y el petroglifo del Museo Nacional (ubicado sobre el espejo de agua en el interior del edificio principal).

Finalmente, la Fundación para el Rescate y la Protección del Patrimonio de la Casa Presidencial suscribe un convenio cada cuatro años con el nuevo Ministerio de la Presidencia con el fin de que sus bienes (la colección de arte, biblioteca, alfombras, vajilla y un sin número de muebles) se encuentren a entera disposición de los nuevos integrantes del gobierno. La Fundación ha sido una entidad cultural independiente, sin fines de lucro y sin filiación política, integrada por un cultural. El 6 de diciembre de 1994, se creó la Fundación por iniciativa de la entonces Primera Dama, la Sra. Josette Altman de Figueres.

\section{El patrimonio cultural inmueble}

La sede de la Presidencia de la República se encuentra ubicada en Zapote, en la ciudad de San José, y alberga el despacho del presidente y las oficinas de su 
gabinete. El edificio principal de la sede del gobierno reúne las características de un patrimonio inmueble ya que éste se enaltece con la arquitectura contemporánea que le imprimió Pedro Ramírez Vásquez, uno de los arquitectos mexicanos más admirados e influyentes del siglo XX.

Para interpretar el valor simbólico de un inmueble arquitectónico, Vázquez Piombo (2009, p. 11) recurre a un modelo metodoloógico que incluye el conocimiento de la historia, la observación del lugar, la indagación formal y las nuevas necesidades y manifestaciones contemporáneas, asi como la herramienta de la hermaneutica que permite develar más

\section{La historia de la sede de la Presidencia}

Con el fin de brindar una síntesis histórica de la sede de la Presidencia es importante destacar que la idea de trasladar la sede del gobierno al edificio en Zapote, nace durante el gobierno del Dr. Rodrigo Carazo Odio (1978-1982). En ese enton-

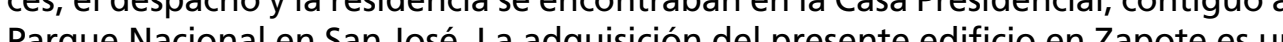

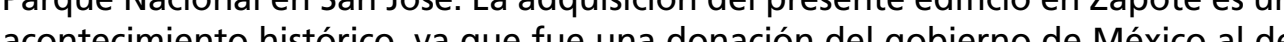
costa Rica, como resultado de las conversaciones entre los entones presidentes costáxico, José López Portillo, y de Costa Rica, Rodrigo Carazo Odio. En una en de México, José López Portilo, y de Costa Rica, Rodrigo Carazo Odí. En una enlrevsista -en la cual me encontraba presente- ex prexidente Carazo compartió co.'Yo le doy un dólar a usted y firmamos'. Nos costó un dólar. Yo saqué un dólar

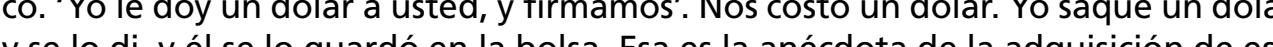
y se lo di, y el se loguardón la bolsa. Esa es la anécdota de la adquisición de es 14 de octubre del 2004).

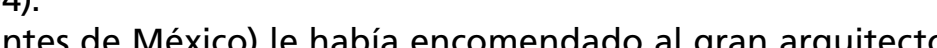
Pedro Ramírez Vázquez la construcción del edificio en 1976. Posteriormutecto Pedricico se convirtió en la construcción del edificio en 1976. Posteriormente, e edificio se convirtió en las oficinas de FERTICA (Fertilizantes de Costa Rica). Finalme 1980 y se denominó Presidencia de la República de Costa Rica y marzo de 1980 y se denomidén Otro detalle que se alberga el despacho yu expresó en su libro Tiempo y marcha en relación a los motivos por los cuales se colocó una placa en la sede de la Presidencia (Carazo, 1989: p. 493) Nunca me he explicado por qué se arrancó la placa que colocamos a la entrada del
edificio, en la que se indicaba la fecha en que la oficina de la Presidencia de la República se trasladó a Zapote, así como que el edificio "fue adquirido por el pueblo"..

Se puede adivinar el motivo por el cual se arranca indebidamente una placa de un edificio público, tratando de borrar el nombre del gobernante que los construyó, pero arrancar placas que solo decían "construido por el pueblo", o "adquirido por el pueblo", sin hacer mención a Carazo por ningún lado y en este caso en especial en la propia Presidencia, es el colmo...

Lamentablemente, esta placa aún no se ha ubicado.

En este breve análisis no se puede incluir un estudio exhaustivo de la historia de la sede de la Presidencia (1980-2013), pero sí cabe destacar dos momentos cuando la sede del gobierno corrió el grave peligro de ser trasladada en los últimos años. En primera instancia, el ex presidente Oscar Arias anunció su interés en trasladar la sed de la Presidencia a las instalaciones del Centro Nacional de Cultura (CENAC) pero, debido a la fuerte oposición del gremio cultural del país, esta decisión no se efectuó (Díaz, 2006). La segunda situación fue cuando el mismo ex presidente anunció, al final de su administración, que había inaugurado el nuevo sitio donde iba a construir la nueva sede de la Presidencia y resaltó lo siguiente: "Ojalá que nos dejen una obra que nos llene de orgullo a los costaricenses. De las casas presidenciales, la de Costa Rica

puede imaginar en Centroamérica" (Rojas, 2011). Lo que preocupa en ambos casos es que, hasta la fecha, no existe evidencia de que la actual sede de la Presidencia Por este motivo, es importante destacar el legado histórico político y cultural de Pe del gobierno para que no se convierta en sujeto de decisiones arbitrarias que pueden conducir a su deterioro, destitución o destrucción.

\section{Arq. Pedro Ramírez Vásquez}

El arquitecto Pedro Ramírez Vázquez es considerado "el padre de la arquitectura moderna de México" (Bautista y Sánchez, 2013) y es responsable de la Construcción de algunos de los edificios más emblemáticos de este pais. El autor Vázquez, acentúa la expresión poética de la obra del arquitecto, Pedro Ramirez taca " la sencillez de sus composiciones, la claridad con que se nos ofrecen sus proy...la sencillez de sus composiciones, la claridad con que se nos ofrecen sus proyectos, la nitidez de su dibujo en el len cor Cuando el Arq. Ramírez Vásquez constrú́ su presificia..."

Cuando el Arq. Ramírez Vasquez construía el edificio para FERTIMEX en 1976 (la actual sede de la Presidencia de la República de Costa Rica), también se encontraba construyendo la Nueva Basilica de Santa María de Guadalupe (México DF) y terminaba de construir más de 150,000 unidades de Aula Casa Rural y Escuelas ya había inaugurado el Museo de Nacional de Antropología (México DF) y el Muya había inaugurado el Museo de Nacional de Antropología (Mexico DF) y el Mu1968 fue el Presidente del Comité Organizador de las XIX Olimpiadas en México y en 1971 construyó el Museo de las Civilizaciones Negras (Dakar, Senegal). El Arq. Ramírez Vázquez participaría en múltiples proyectos a lo largo de su vida en el cual también se destaca el Museo de la Olimpiadas (Lausana, Suiza) en 1986.

Las características del edificio

De acuerdo la semiótica arquitectónica, Eco $(1986$, p. 268) recurre a los conceptos de función primaria, para referirse a las funciones de denotación (significante) y a la función secundaria, para referirse a las funciones connotativas (significado). Para el autor, las expresiones "primaria" y "secundaria" no tienen un valor discriminativo en un sentido axiológico, o sea, que una fuera más importante que la otra, sino que desea expresar que las funciones secundarias se apoyan en las 
primarias. De esta forma, en la sede de la Presidencia podemos clasificar como funciones primarias y secundarias los siguientes conceptos expresados por el Arq Pedro Ramírez Vázquez, en una ficha técnica elaborada en 1976, la cual brindó la Fundación para el Rescate y la Protección del Patrimonio de la Casa Presidencial:

"Este edificio consta de dos grandes cuerpos que enmarcan un patio central con un enorme espejo de agua. La cubierta es de un material translúcido que favorece un clima de luz, humedad y vegetación que es propio de la arquitectura tropical. Los dos cuerpos del edificio están vinculados por medio de rampas. Por estar a medios niveles uno del otro, se pueden disfrutar de grandes perspectivas a través de los propios salones de las oficinas. La aportación más importantes es la integración espacial entre el patio, el vestíbulo y el paisaje en un ambiente que

\section{El patrimonio cultural mueble}

Gran parte del patrimonio mueble pertenece a la Fundación para el Rescate y la Protección del Patrimonio de la Casa Presidencial y constituye uno de los intereses primordiales de esta organización.

La biblioteca

La Fundación tuvo la iniciativa de conformar una biblioteca en la sede de la Presidencia con el fin de preservar y difundir el patrimonio documental. Gracias al apoyo de la Academia Costarricense de la Lengua, la Editorial de la Universidad de Costa Rica y diversas editoriales nacionales, así como autores de la sociedad académica y civil, se recopiló una colección importante de libros para conformar la biblioteca de la Fundación. La colección comprende textos de historia, geografía, política y literatura relacionados a la cultura costarricense.

La colección de arte

La colección de arte de la Fundación ha sido posible gracias a la inmensa generosidad de los artistas costarricenses e internacionales y a las invaluables gestioy curadores de arte voluntarios de la Fundación. Este patrimonio artístico reún y cús de 250 pinturas, esculturas, dibujos, grabados y fotografías de las últimas mácadas del siglo XX, y todas fueron donadas por los artistas. Todas las ulltimas decadas del siglo XX, y todas fueron donadas por los artistas. Todas las obras de despachos de la sede de la Presidencia.

La colección de arte de la Fundación también tiene el honor de contar con el mural Reminiscencias Indígenas realizado por la artista costarricense Lola Fernández, premio Magón 1995. Este mural fue inaugurado durante la visita de Sr. Álvaro Uribe, presidente de Colombia, el 19 de junio de 2003 . Este mural se ejecutó gracias al generoso a Colombia, el tante colaboradora de la Fundación.

\section{El petroglifo}

La sede de la presidencia cuenta con un petroglifo precolombino, custodiado por el Museo Nacional. La Fundación le solicitó al museo realizar los estudios correspondientes para brindar la información necesaria a los visitantes, y los resultados de estos estudios reflejan que el petroglifo había sido hallado cerca de Colonia Blanca de Upala, provincia de Alajuela. El traslado del petroglifo se realizó en 1981 por el Sr. Rodrigo Carazo Odio, entonces Presidente de la República, quien lo encontró removido de su lugar original y en peligro de ser sustraído de manera ilegal.

\section{Los muebles y varios}

La Fundación para el Rescate y la Protección del Patrimonio de la Casa Presidencial ha invertido en la compra de muebles, alfombras y vajilla, con el fin de brindarle a sus visitantes.

\section{El patrimonio de la sede de la presidencia: problemas y soluciones}

"Esta es la casa de todos los costarricenses" es el texto que recibe a todos los visitantes en la entrada principal de la sede de la Presidencia de la República de Costa Rica; sin embargo, este mensaje suele pasar desapercibido o resultar contradictorio para la mayoría de costarricences que aún no han tenido acceso a este espacio. Uno de los motivos por los cuales se generan situaciones de inclusión y exclusión es debido a las estrictas medidas de seguridad que se mastienen en este recinto. Sin embargo, latro motivo radica en que la sede de la Presidencia de la República ha descuidado su papel icención como una instancia promotora de

La segunda situación que resulta problemática, es que el edificio de la sede de la Presidencia aún no ha sido declarado patrimonio nacional por las fuentes oficiales que han conducido a su desvaloración arquitectónica y deterioro físico. Este tipo qu intervenciones se debe, en gran medida, al gran desconocior

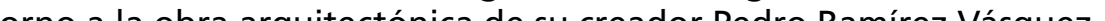

El terce, y ultimo panorama que resulta muy preos que se encuentran los bienes culturales tangibles e intangibles de la Presidencia. A esar de que las diversas administraciones guntribuido a vede por sus activos, y que La Fundación para indispensable para la colección y custodia de sus bienes, ambas instancias han carecido de gestiones administrativas que dia de sus bienes, ambas instancias han carecido de gestiones administrativas que incluyen programas de documentación, restauración y difusion educativa

es importante destacar la reciente desintegracion de esta Fundación. jurídica de los bienes culturales de la Presidencia, el conjunto de estos recursos simbólicos tienen la necesidad de recurrir a conceptos y prácticas que son implementadas actualmente en los campos del patrimonio y la museología. Por ejemplo, la estructura museológica -teórica y práctica- ofrece una serie de beneficios: en primer lugar, le otorga una mayor protección a los bienes tangibles, principalmente ante situaciones relacionadas al deterioro, la restauración, el robo o el tráfico ilítito; en segundo lugar, brinda las pautas necesarias para la adquisición y/o la recuperación de nuevas expresiones culturales; $y$, en tercer lugar, facilita las gestiones pertinentes a la exhibición y a la difusión de la colección de sus bienes culturales. 


\section{Bibliografía}

Bautista, V. y Sánchez, L.C. (1 de abril de 2013). Fallece el arquitecto Pedro Ramírez Vázquez. Excélsior. Dirección: http://www.excelsior.com.mx/comunidad/2013/04/01/894305. Consulta: 9 de agosto de 2015.

Benavides Barquero, M. (Enero-Diciembre, 2005). Relación entre Estado e Iglesia en Costa Rica a través de su historia. En: Revista de Historia de América. Núm. 136: pp. 103-128.

Carazo, R. (1989). Tiempo y marcha. Costa Rica: EUNED.

Díaz, D. (31 de mayo de 2006). Artistas y funcionarios reavivan lucha por CENAC. La Nación. Dirección: http://wvw.nacion.com/ln_ee/2006/mayo/31/aldea0.html Consulta: 9 de agosto de 2015.

Eco, U. (1986). La estructura ausente. Introducción a la semiótica. España: Editorial Lumen. Dirección: file:///Users/kirstengrunberg/Downloads/eco_laestructuraausente.pdf Consulta: 9 de agosto de 2015.

Garia Torrego, J. (2011). Actualización del concepto Aura. Antroposmoderno. Dirección: http://webcache.googleusercontent.com/search?q=cache:Qi4avfJU0-4J:www.antroposmoderno.com/antro-articulo.php\%3Fid_articulo\%3D1288+walter+benjamin+con cepto+aura \&cd=5\&hl=en\&ct=clnk\&client=safari\&source=www.google.com. Consulta: 9 de agosto de 2015 .

Gentile, E. (2006). Politics as Religion. USA: Princeton University Press.

González, L.A. (2003). Estética de la arquitectura contemporánea. Un enfoque desde la filosofía relacional (Tesis doctoral). Universidad Complutense, España. Dirección: http://eprints.ucm.es/4789/. Consulta: 9 de agosto de 2015.

Ministerio de Cultura y Juventud. (2012). Patrimonio Cultural. Propuesta de la Ley General de Cultura Costa Rica. Dirección: https://web.archive.org/ web/20121117015026/http://politica.cultura.cr/propuesta/1-patromonio-cultural. Consulta: 9 de agosto de 2015.

Quijano, A. (2000). Colonialidad del poder, eurocentrismo y América Latina en La colonialidad del saber: eurocentrismo y ciencias sociales. Perspectivas latinoamericanas. Edgardo Lander (Compilador). Buenos Aires: Consejo Latinoamericano de Ciencias Sociales CLACSO.

Rojas, R. (3 de mayo, 2011). Arias develó placa de futura Casa Presidencial en un parqueo. La Nación. Dirección: http://www.nacion.com/2010-05-04/ElPais/UltimaHora/ElPais2359636.aspx. Consulta: 9 de agosto de 2015.

Vargas Salguero, R. (1995). Pabellones y museos de Pedro Ramírez Vázquez. México: Editorial Limusa.

Vázquez Piombo, P. (2009). Arquitectura contemporánea en contextos patrimoniales: una metodología de integración. México: Instituto Tecnológico y de Estudios Superiores de Occidente. 